\title{
Designing an Efficient Ultra Small Form Factor On-Chip Antenna for UHF RFID Application
}

\author{
Balazs MATOLCSY, Attila ZOLOMY \\ Dept. of Broadband Infocommunication and Electromagnetic Theory, \\ Budapest University of Technology and Economics, Egry Jozsef, 3, 1111 Budapest, Hungary \\ matolcsy@hvt.bme.hu, zolomy@hvt.bme.hu \\ Submitted December 3, 2018 / Accepted March 17, 2019
}

\begin{abstract}
The efficient design of electrically small antennas (ESA) has recently evolved to an outstandingly active area of research. This paper aims to present an improved performance electrically small on-chip antenna design, for the $920 \mathrm{MHz} U \mathrm{HF}$-RFID frequency. Performance improvement is based on creating a slow-wave structure with threedimensional spiral meandering and dielectric loading of the complete antenna structure. A single layer spiral on-chip antenna simulation is presented first, based on a commercially available product, then an improved single layer spiral antenna and finally an alternative 3D multi-layer spiral on-chip antenna is investigated. The proposed alternative on-chip antenna structure fills a $2.5 \times 2.5 \times 1.3 \mathrm{~mm}^{3}$ volume and has significantly improved radiation performance compared to the single layer solution.
\end{abstract}

\section{Keywords}

On-chip antenna design, antenna design, electrically small antennas (ESA), UHF RFID tag design

\section{Introduction}

Modern portable telecommunication devices are tending to exhibit reduced physical dimensions, however the excessive demand for increasing computational power and highspeed data transmission require innovative integration solutions. High speed data rates often require higher operational bandwidth, better performing complex antenna systems and efficient power management methods. One of the key aspects to design modern telecommunication devices (such as smartphones and other wearable devices) is to create well performing multi-band integrated antenna systems [1] in a highly restricted space. This trend is enforcing antenna designers to create reduced size antennas (not necessarily in all dimensions), such as planar printed antennas [2] (e.g. printed inverted-F antenna, inverted-L antenna etc.) or integrated on-chip antennas [3]. This significant demand for antenna downsizing (i.e. miniaturization of antennas, or reducing antenna dimensions) has risen excessively in the past 30 years. There are a number of applications (e.g.: RFID tags) where this miniaturization is exceptionally important [4]. For example in case of wearable devices, or small scale integrated solutions. Many of these applied miniaturization approaches utilize bended [5], meandered antenna designs [6], or booster antennas [7]. However these procedures are only utilizable if the antenna's physical dimensions can be in the range of the operating wavelength [8]. This paper discusses another approach where the RFID antenna is placed on the chip itself, hence the name (on-chip). This design method requires a different approach for determining antenna performance and radiation parameters in the simulation domain. Although, there is a well-formed measurement solution for measuring antenna radiation pattern for UHF RFID antennas, presented [9], obtaining the appropriate antenna parameter results in simulation domain for such ultra small scale antennas require further research (as presented in Sec. 3.3). The rest of the paper is organized as follows: Section 2 discusses miniaturization techniques, limitations and the major drawbacks of antenna downsizing. In Sec. 3 the passive RFID (Radio-Frequency Identification) technology is summarized and the passive UHF RFID tag antenna design practice is discussed. In Sec. 4 the reference design simulation setup and simulation results are presented. The improved reference antenna structure is presented afterwards in Sec. 5. Later on in Sec. 6 the proposed three-dimensional spiral antenna design is shown and the results are presented. Finally in the last section the paper is concluded.

\section{Main Aspects of Antenna Downsizing}

In this section the practice, the purpose, the beneficiary effects and drawbacks of the downsizing procedure is presented, starting out with a major definition problem: when is it appropriate to describe an antenna as small? 


\subsection{Definition of Small Antennas}

The most common used expression for small antennas in scientific papers is: Electrically Small Antennas (ESA). This refers to the fact, that the best practice is to compare antenna physical dimensions to the operating wavelength $(\lambda)$ of the antenna. A common definition for an ESA is having dimensions in the range:

$$
k a \leq 0.5
$$

where parameter $k=2 \pi / \lambda$ and parameter $a$ is the half-length of a thin linear dipole [11]. There is another version of this definition where $a$ is the radius of a sphere surrounding the antenna structure. An important subset of ESA's are the so called Physically Constrained Small Antennas (PCSA). A PCSA is an antenna, that has at least one dimension which fulfills the ESA dimension criteria set in (1). An adequate example for this is an inset patch antenna in the X-Y plane, having much smaller dimensions compared to the operating wavelength in the Z-direction (e.g. in the range of $\lambda / 50$ ). A Physically Small Antenna (PSA) only refers to the physical dimensions of the antenna, regardless of the operation frequency. The rest of this paper discusses ESA's having dimensions, all of which complies with the aforementioned definition criteria.

\subsection{The Purpose of Downsizing}

The concept of antenna downsizing spread at an unexpected rate, as telecommunication devices began to shrink in terms of physical dimensions. Handheld and miniature platforms require efficient design of electrically small antennas. The smaller the volume occupied by the antennas from the space inside or on the device, the better. This enforces antenna design engineers to sacrifice antenna performance for antenna size. The general purpose of downsizing (i.e. miniaturization) is to maintain antenna radiation properties and performance as well as possible, while decreasing physical dimension(s) at the same time. The straightforward solution would be the linear downscaling of antenna dimensions. This would result in uneven current distribution, reduced radiation efficiency, higher resonance frequency, etc.; in short: dramatically decreased overall antenna performance. Therefore efficient downsizing requires a range of special geometry and material transformations.

\subsection{Principles of Downsizing}

The downsizing process (i.e. construction of an ESA), can happen in many different ways, but every method aims one (or more) of these fundamental goals [12]:

- lowering the resonance frequency for an antenna with fixed dimensions;

- efficiently filling the space circumscribing the largest dimension of the antenna;

- creating a conjugate complementary antenna structure.
An adequate way of lowering the resonance frequency of an antenna (while maintaing all physical dimensions) is to compose the antenna with a slow-wave structure. In a slow-wave structure, the electromagnetic wave's propagation speed $v_{\mathrm{p}}$ is lower than the speed of light $\mathrm{c}$. This implies that an antenna composed with a slow-wave structure will exhibit lower resonance frequency. A common technique for designing a slow-wave structure is to modify the antenna geometry, in order to extend the high frequency current path. This extension can be a meandered line structure [13], a fractal structure [14], slits in a metal plane, etc.

Not only do these solutions lower the resonance frequency, but if properly designed they also fill the space dedicated to the antenna structure efficiently. There are some planar geometry structures (e.g Peano [15] and Hilbert [16] curves, depicted in Fig. 1 and Fig. 2), which efficiently fill the largest available planar space.

Antennas composed of conjugate component structures are also promising candidates for realizing efficient electrically small antennas (example seen in Fig. 3). The theory of operation behind complementary structures, is that their capacitive and inductive components partially compensate each other, thus resulting in better radiation performance and relatively frequency independent behaviour. This phenomena had been investigated by Hohlfeld and Cohen based on the work of Rumsey. The self-complementarity and origin symmetry requirements form together the frequency independent behaviour in antennas [17].

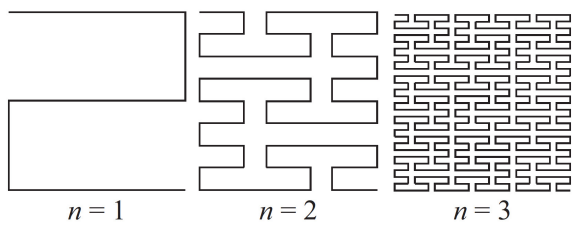

Fig. 1. First, second and third-order Peano curves.

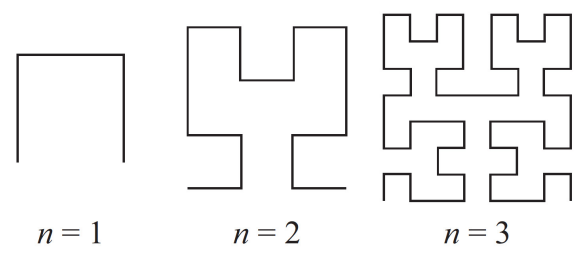

Fig. 2. First, second and third-order Hilbert curves.

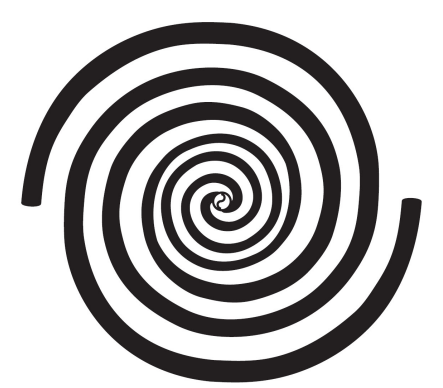

Fig. 3. Self-complementary planar spiral antenna structure. 


\subsection{The Drawbacks of Antenna Downsizing}

Contrary to the efforts made to attain antenna performance by employing proper downsizing methods, miniature antennas will most likely suffer from at least some of the following disadvantageous properties:

- reduced radiation efficiency;

- distorted and degraded radiation pattern;

- narrow relative bandwidth, high quality factor $(Q)$.

Apart from the above, a key property of the antenna always decreases during the downsizing process, which is the effective antenna length. This parameter is in close relation to the amount of electromagnetic (EM) energy that can be extracted from the EM field surrounding the antenna. The larger the effective length is, the better the extraction efficiency is. In case of ultra-small form factor antennas (where $k a \leq 0.5$ ) it's a reasonable practice to describe antenna performance with quantities in close relation to the effective length (e.g. power flow).

\section{Electrically Small, Passive UHF RFID On-Chip Antenna}

\subsection{Passive RFID Systems}

RFID is a wireless technology used to transmit digital data between stationary devices (commonly referred to as: readers), and one or more moving objects (referred to as: tags). RFID links operate in a wide range of frequency bands [10]. This paper focuses on the $920 \mathrm{MHz}$ UHF RFID frequency. The expression passive in RFID systems refers to an RFID tag that lacks internal power source, thus the tag gains energy from the rectified power of the incident absorbed EM wave. This rectification process takes place inside the CMOS chip, which is attached to the RFID tag antenna. The communication process in a passive RFID link is the following.

The reader transmits EM power towards the passive tag's antenna, providing enough power for the tag chip to start the operation. The chip processes the incoming data and sends the data requested by the reader. The transmission happens by periodically changing the output impedance of the tag chip (in accordance with the transmitted data), thus either reflecting, or absorbing the incident EM wave generated by the reader. This principle is similar to other reflection based detection techniques and it was first introduced by H. Stockman [18] who described it as: "Communication by means of reflected power". This type of transmission is not designed for high data rates, thus the data speed is not necessarily the most important quantity of RFID communication. The crucial property of a passive RFID link, is the largest available transmission distance (between the tag and the reader). Since this quantity vastly depends on the available transmit power of the reader and performance of the tag antenna, hence the following sections of this paper focus on the methods of improving the tag antenna performance.

\subsection{Ultra-Small Form Factor UHF RFID Tag}

The idea of the antenna tag design for further simulations is based on a commercially available RFID tag chip. The Ultra Small Package Tag (USPT) by Hitachi Chemical Co. Ltd. is a complete passive RFID tag with an Impinj Monza 5 CMOS chip and integrated onchip antenna. Regarding physical dimensions, the tag itself is $2.5 \times 2.5 \times 0.4 \mathrm{~mm} \mathrm{[19]} \mathrm{and} \mathrm{the} \mathrm{operating} \mathrm{frequency} \mathrm{is}$ $920 \mathrm{MHz}$. The on-chip antenna on the device complies with the definition of being electrically small. The parameter $k a$ is calculated with the spherical condition introduced in Sec. 2.1.

$$
k a=\frac{2 \pi}{\lambda} a \approx \frac{6.28 \cdot 3.54 \cdot 10^{-3}}{0.326} \approx 0.068 .
$$

Since (1) is fulfilled in (2), the antenna is considered electrically small. The antenna structure (depicted in Fig. 4) is an orthogonally designed planar spiral loop antenna. Unfortunately the manufacturer does not provide public, reliable simulation and measurement results related to the on-chip antenna performance, therefore the simulation design presented here is based on the values found in the datasheet, which is accessible on the manufacturer's website [19].

The manufacturer provides application notes for designing booster antennas for the device, however adding a booster structure would increase the overall physical size of the device. Our aim is to optimize the on-chip antenna performance by utilizing different antenna compositions, without the significant increment in antenna dimensions. The first antenna simulation is based on the idea of the USPT tag on-chip antenna.

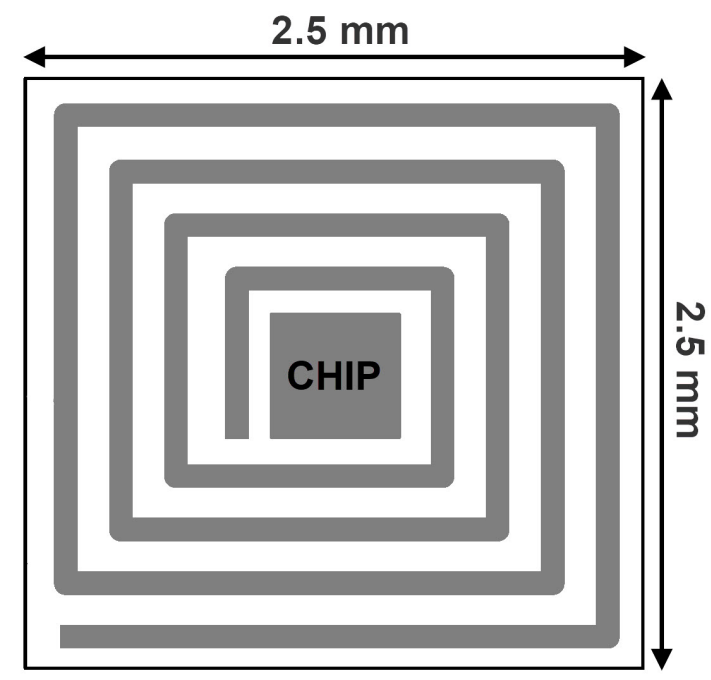

Fig. 4. Top view of the USPT with on-chip antenna [19]. 


\subsection{Simulation of the Ultra Small Package Tag}

For simulation CST Microwave Studio 2018 was used. The three-dimensional model of the USPT is illustrated in Fig. 5. The model lies in the X-Y plane. Due to the ultrasmall form factor, the bounding box and model meshing played a key role at simulation accuracy. Bounding box planes are translated in all directions $(\mathrm{X} ; \mathrm{Y} ; \mathrm{Z})$ with a distance: $\lambda / 10$ from the simulation model (open boundary conditions were set on all box planes). The discrete port excitation of the antenna structure is located on the bottom side of the chip at the endpoints of the spiral. The RFID chip manufacturer (Impinj) provides a wideband circuit model (shown in Fig. 6) for the chip's RF output impedance, this is considered in the discrete port parameters. In this construction the on-chip antenna is terminated with the circuit in Fig. 6.

Since the antenna is terminated in the circuit model in Fig. 6, this load is modeled as a lumped element, directly attached to the discrete port. The built-in FR-4 model $\left(\varepsilon_{\mathrm{r}}=4.5, \tan \delta=0.025\right)$ was utilized as a substrate (unfortunately the manufacturer does not provide exact substrate parameters for the USPT tag, hence this assumption). The top copper layer width was $35 \mu \mathrm{m}$ and modeled as a Perfect Electric Conductor (PEC). CST Microwave Studio offers a frequency domain solver (F-solver), which is commonly used for high frequency problems. This F-solver was used to obtain simulation results.

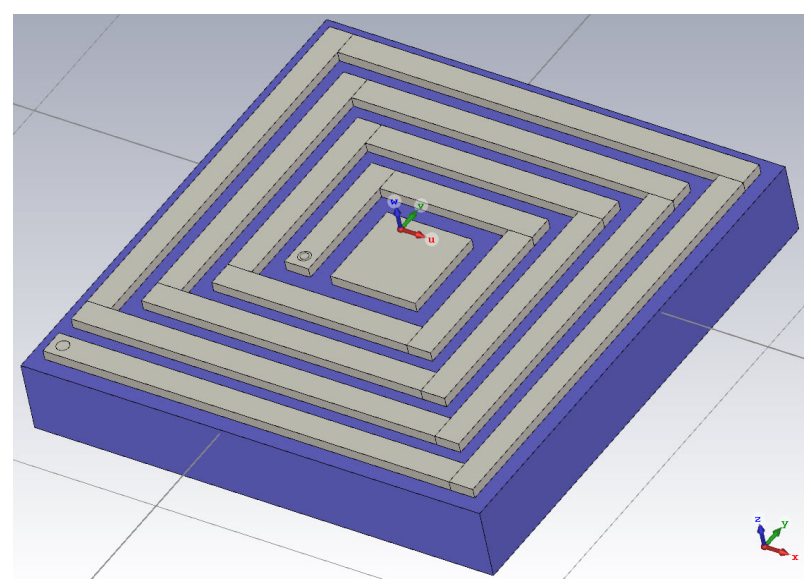

Fig. 5. 3D model of the USPT in Microwave Studio 2018.

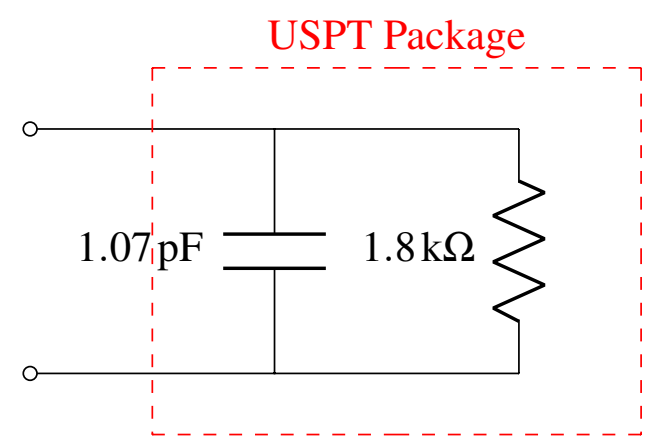

Fig. 6. USPT circuit model.
The on-chip antenna performance was evaluated by the bulit-in power flow field monitor in CST. The power flow monitor calculates the magnitude of the absolute value of the Poynting vector on a dedicated curve, or a face above the antenna structure. Instead of the far-field monitors, it's an advantageous practice to use a power flow monitor in this situation. Due to the extreme size ratio between antenna dimensions and operating wavelength, a major problem arises. Accurate far-field calculations require a bounding box with planes at least $\lambda / 2$ apart from the structure, which would result in a huge volume calculation domain with inherently dense meshing, hence extreme computational complexity. For this reason, far-field calculations were omitted. Furthermore, during the RFID transmission in such small sized onchip antennas, the energy for the passive RFID tag is coupled through the near-field inductive component of the reader's antenna. For this reason, near field dependent quantities are used for adequate antenna performance comparison.

\section{Reference Design Simulation}

Figure 7 shows the amount of power flow, purpendicular to the $\mathrm{X}-\mathrm{Y}$ plane, at $Z=5000 \mu \mathrm{m}$. For an exact comparison, two cutting planes were applied and the power flow amount was evaluated along the $\mathrm{X}$ and $\mathrm{Y}$ axis (denoted with dashed black lines on Fig. 7). These cutting plane values are depicted in Fig. 8 and Fig. 9. These simulation results show that, the absolute maximum value of the power flow is approximately $250 \mathrm{VA} / \mathrm{m}^{2}$. This power flow quantity is set, as a reference. The antenna input impedance $\left(Z_{\text {in }}\right)$ was calculated at the excitation port, resulting in $Z_{\text {in }}=41-2300 \mathrm{j} \Omega$ and the input reflection coefficient is: $S_{11}=-0.096 \mathrm{~dB}$. This former impedance value is far from the ideal pure real $1.8 \mathrm{k} \Omega$, thus the antenna should be tuned.

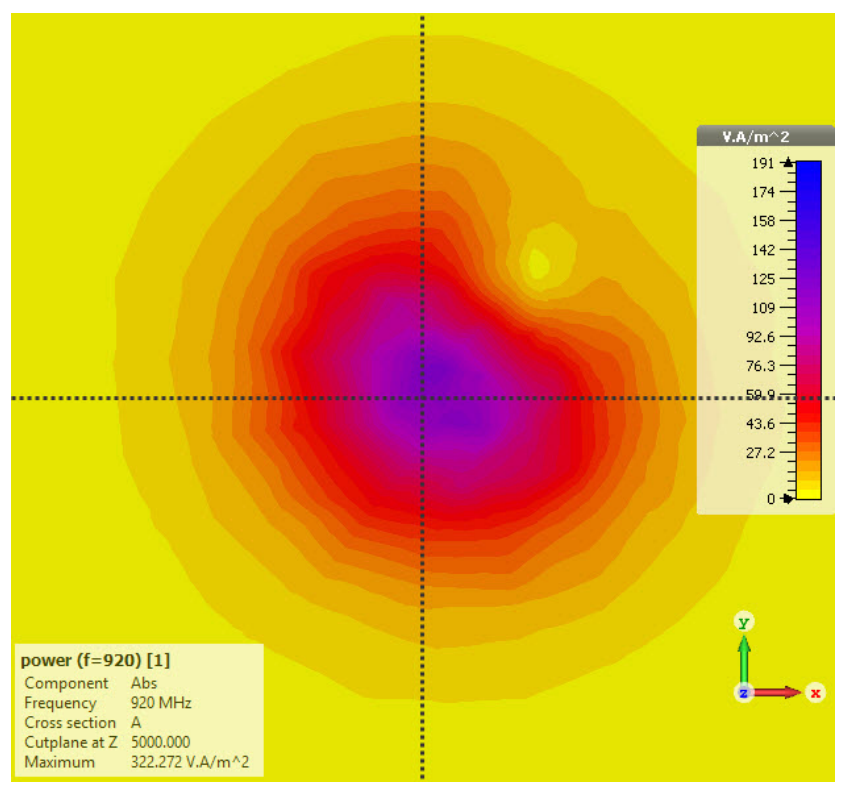

Fig. 7. Power flow parallel to the $X-Y$ plane, at $Z=5000$. 


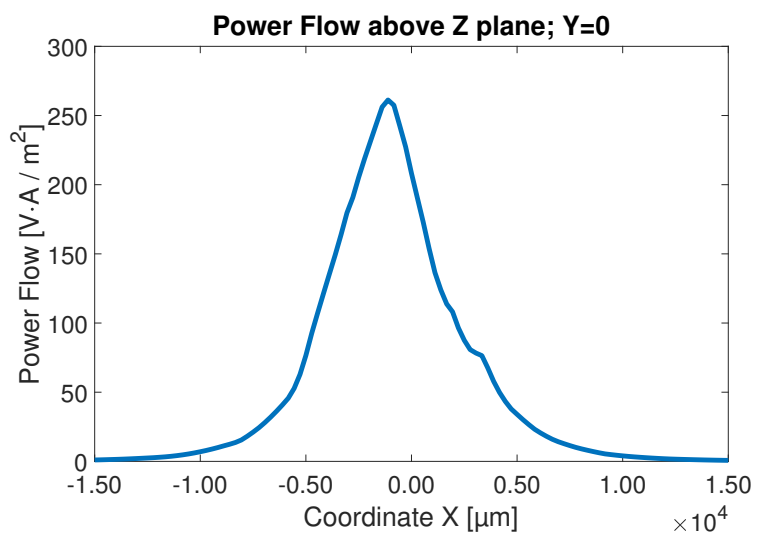

Fig. 8. First antenna performance, $x$-axis.

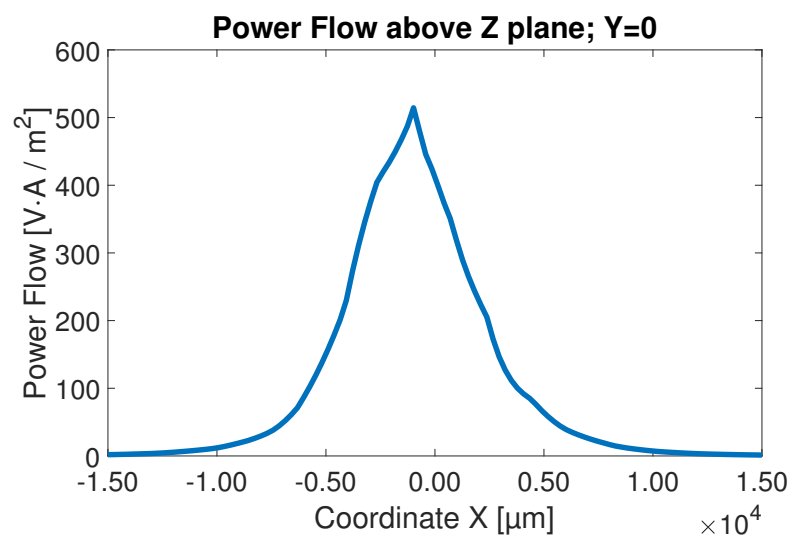

Fig. 10. Modified antenna performance, $\mathrm{PEC}=10 \mu \mathrm{m}$, $\mathrm{x}$-axis.

\section{Improving the Reference Design}

From the parametric sweeps applied to the first model, it was clear that the antenna performance mainly depends on the width of the top PEC layer. Figures 10 and 11 show the results where modified $10 \mu \mathrm{m}$ width PEC layer was chosen. By adjusting the layer width, the modified antenna performance (power flow) significantly improved, to a maximum value of $500 \mathrm{VA} / \mathrm{m}^{2}$. Decreasing the PEC layer width causes smaller distributed capacitance along the meandered planar structure, leading to partial structural resonance and better performance. The same results were present when the original PEC width and a reduced lumped capacitance value was inserted $(0.9 \mathrm{pF}$ instead of $1.07 \mathrm{pF})$. The modified antenna impedance changed. The modified antenna has a $Z_{\text {in }}=1330-12200 \mathrm{j} \Omega$. The real part of the antenna impedance improved significantly, hence the better antenna performance. Input reflection coefficient is: $S_{11}=-0.27 \mathrm{~dB}$. The power flow almost doubled, compared to the original design. This improvement is related to the reduced metallic layer (PEC). Another approach for increasing the antenna performance was to change the substrate material, in order to create a slow-wave structure, however, no further improvements could be reached by simply changing the single layer substrate material parameters.

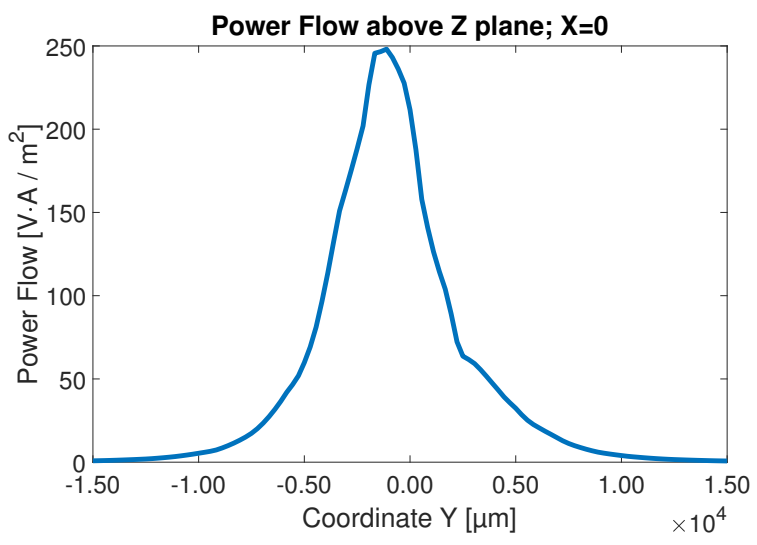

Fig. 9. First antenna performance, y-axis

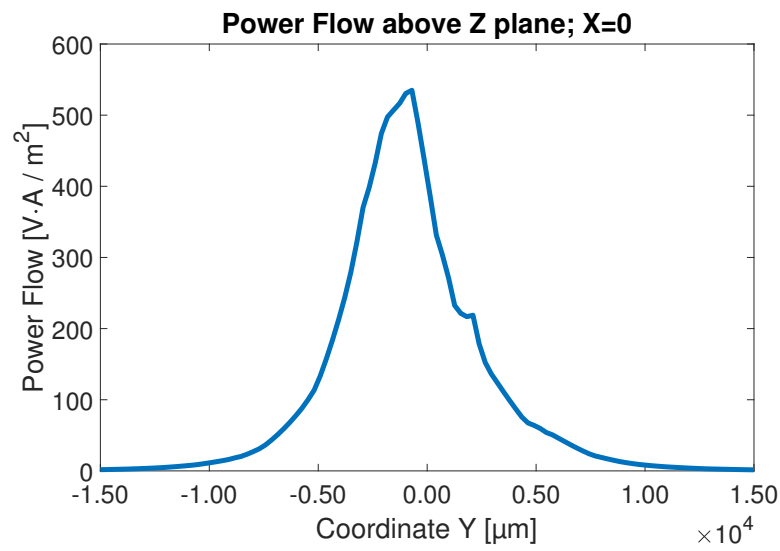

Fig. 11. Modified antenna performance, $P E C=10 \mu \mathrm{m}$, y-axis.

\section{An Alternative On-Chip Antenna}

Based on the promising results of the previous spiral antenna, a customized three-dimensional multi-layer ceramic spiral antenna was designed. The base size of the antenna was identical, but the substrate width was increased to three layers $(300 \mu \mathrm{m}$ each), containing an evolving internal spiral. The new antenna followed the previous spiral structure with an elevation in the direction of the $\mathrm{Z}$ axis. After each complete round the spiral continues above the previous layer. This antenna structure is depicted in Fig. 12. The transparent solid surrounding the $3 \mathrm{D}$ spiral structure is the changed substrate with a dielectric constant $\varepsilon_{\mathrm{r}}=20$. The input reflection coefficient is $S_{11}=-1.56 \mathrm{~dB}$ and $Z_{\text {in }}=896-3759 j \Omega$. Since the input reflection coefficient improved significantly, our expectations would forecast an increased amount of power flow. The power flow was evaluated at the same position above the antenna planes as in Sec. 4.

The simulation results are shown in Fig. 13 and Fig. 14. The antenna performance has improved, the power flow maximum reached $700 \mathrm{VA} / \mathrm{m}^{2}$.

This three-dimensional spiral shaped antenna type could be a well-formed alternative to the first presented onchip antenna, as this antenna exhibits better performance 
without major size increment in planar dimensions. This alternative antenna structure has almost three-times better performance than the first simulated antenna, in terms of power flow. Opting for this alternative antenna design leads to increased reading distance for RFID tags, without the need for higher transmitted power from the reader source. The relationship between reading distance and power-flow is subject to further research.

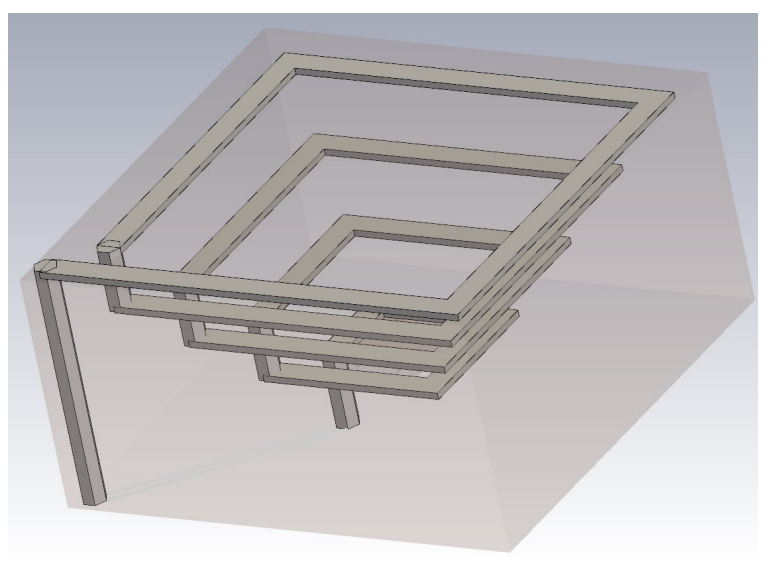

Fig. 12. 3D spiral antenna model, perspective-view.

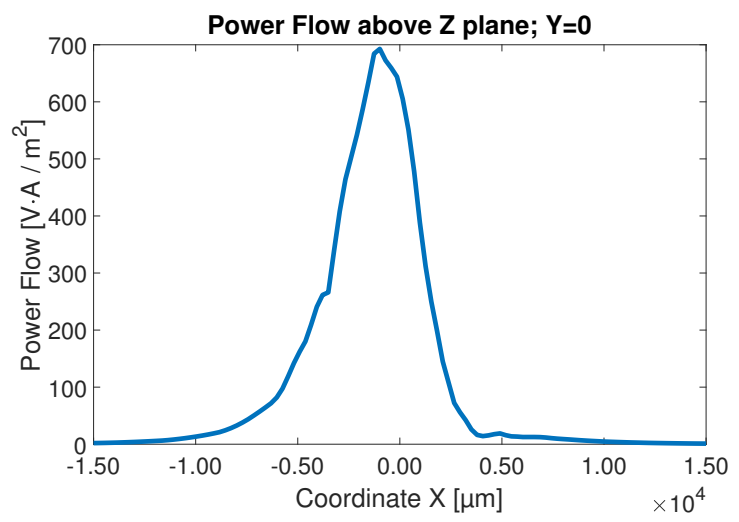

Fig. 13. 3D multi-layered spiral antenna power flow, $x$-axis.

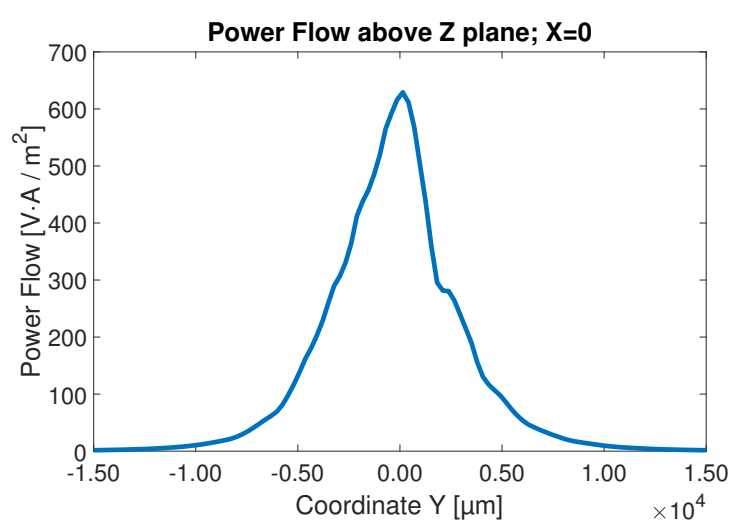

Fig. 14. 3D spiral antenna power flow, y-axis.

\section{Future Work and Recent Results}

Based on the promising results of the simulated multilayer antenna structure in Sec.6 a test sample is under construction. The proposed multi-layer ceramic structure is going to be manufactured with LTCC (Low Temperature Cofired Ceramic) technology, similarly to the multi-layer helical antenna presented in [20]. Measurement comparison of the commercially available product (USPT Tag by Hitachi) and the proposed multi-layer antenna structure is also planned in the future. Ultra-small scale antennas are an active area of research as the demand for small IoT devices tend to rise. The latest papers in this area of research consistently try to push the antenna dimensions downwards, as mentioned in [21] and in [22] as well.

\section{Conclusion and Summary}

This paper introduced on-chip antenna structures used is UHF-RFID systems for $920 \mathrm{MHz}$. An alternative threedimensional multi-layer spiral on-chip antenna structure was presented, which exhibited increased performance (higher power flow, and better impedance matching) compared to the first single layer spiral antenna. The multi-layer 3D antenna structure was analyzed using 3D EM software (CST Studio 2018). Alongside with the antenna structure, an alternative quantity is proposed for evaluating antenna performance (due to the issues of such small antenna structures). Based on the promising simulation results the test antenna is going to be manufactured and measured to validate the simulation results.

\section{Acknowledgments}

The authors would like to thank the Deutsche Akademischer Austausch Dienst (DAAD) for financially supporting the research, at the Karlsruhe Institute of Technology (KIT), at the department of IHE (Institut fur Hochfrequenz und Elektrotechnik). I would like to thank for the helpful advice of Dr. Zsolt Kollar (BME) and Dr. Thomas Zwick (KIT).

\section{References}

[1] HSIAO, H., WU, J., LU, J., et al. Multi-band dual-meander-line antenna for mobile handsets. In IEEE Antennas and Propagation Society International Symposium. Albuquerque (USA), 2006, p. 4705-4708. DOI: 10.1109/APS.2006.1711690

[2] AHMED, I., SHOAIB, I., SHOAIB, N., et al. A printed hybrid loop planar inverted-F antenna for next generation handheld terminals. In 7th European Conference on Antennas and Propagation (EuCAP). Gothenburg (Sweden), 2013, p. 2044-2047. ISBN: 9788890701832

[3] TIMOShENKO, A., LOMOVSKAVA, K., SUSLOV, M. Features of design, implementation, and characterization of on-chip antennas for microwave frequencies. In East-West Design and Test Symposium (EWDTS). Rostov-on-Don (Russia), 2013, p. 1-5. DOI: 10.1109/EWDTS.2013.6673081 
[4] DUBOK, A., SMOLDERS, A. B. Miniaturization of robust UHF RFID antennas for use on perishable goods and human bodies. IEEE Antennas and Wireless Propagation Letters, 2014, vol. 13 , p. 1321-1324. DOI: 10.1109/LAWP.2014.2337051

[5] LUK, W. T., YUNG, K. N. Bending dipole design of passive UHF RFID tag antenna for CD / DVD discs. In AsiaPacific Microwave Conference. Macau (China), 2008, p. 1-4. DOI: 10.1109/APMC.2008.4958093

[6] BRAATEN, B. D., REICH, M., GLOWER, J. A Compact meanderLine UHF RFID tag antenna loaded with elements found in right/left-handed coplanar waveguide structures. IEEE Antennas and Wireless Propagation Letters, 2009, vol. 8, p. 1158-1161. DOI: 10.1109/LAWP.2009.2034990

[7] PACHLER, W., BOSCH, W., HOLWEG, G., et al. A novel booster antenna design coupled to a one square millimeter coil-on-chip RFID tag enabling new medical applications. In European Microwave Conference. Nuremberg (Germany), 2013, p. 1003-1006. DOI: 10.23919/EuMC.2013.6686829

[8] JANKOWSKI-MIHULOWICZ, P., KAWALEC, D., WEGLARSKI, M. Antenna design for semi-passive UHF RFID transponder with energy harvester. Radioengineering, 2015, vol. 24, no. 3, p. 722-728. DOI:10.13164/re.2015.0722

[9] JANKOWSKI-MIHULOWICZ, P., WEGLARSKI, M. A method for measuring the radiation pattern of UHF RFID transponders. Radioengineering, 2016, vol. 23, no. 2, p. 163-172. DOI: $10.1515 / \mathrm{mms}-2016-0018$

[10] GS1 EPC ${ }^{\mathrm{TM}}$ Radio-Ferquency Identity Protocols Generation-2 UHF RFID, Specification for RFID Air Interface, Protocol for Communications at $860 \mathrm{MHz}-960 \mathrm{MHz}$, Version 2.0.1 Ratified

[11] KING, R. W. P. The Theory of Linear Antennas. Harvard University Press, 1956. ISBN: 978-0-674-18218-9

[12] FUJIMOTO, K., MORISHITA, H. Modern Small Antennas. Cambridge University Press, 2013. ISBN: 978-0-521-87786-2

[13] RASHED-MOHASSED, J., MEHDIPOUR, A., ALINKBARIAN, H. New scheme of size reduction in space filling resonant dipole antennas. In European Conference on Antennas and Propagation (EuCAP). Berlin (Germany), 2009, p. 2430-2432. ISBN: 978-1-4244-4753-4

[14] BALIARDA, C. P., ROMEU, J., CARDAMA, A. The Koch monopole: A small fractal antenna. IEEE Transactions on Antennas and Propagation, 2000, vol. 48, no. 11, p. 1773-1781. DOI: $10.1109 / 8.900236$

[15] ZHU, J., HOORFAR, A., ENGHETA, N. Peano antennas. IEEE Transactions on Antennas and Propagation Letters, 2004, vol. 3, p. 71-74. DOI: 10.1109/LAWP.2004.827899

[16] CHEN, X., SAFAVI, S., LIU, Y. A down-sized printed Hilbert antenna for VHF band. In IEEE Antennas and Propagation Society International Symposium. Columbus (USA), 2003, p. 581-584. DOI: 10.1109/APS.2003.1219304
[17] HOHLFELD, R. G., COHEN, N. Self-similarity and the geometric requirements for frequency independence in antennae. Fractals, 1999, vol. 7, no 1., p. 79-84. DOI: 10.1142/S0218348X99000098

[18] STOCKMAN, H. Communication by means of reflected power. Proceedings of the Institute of Radio Engineers (IRE), 1948, vol. 36, no. 10 , p. 1196-1204. DOI: 10.1109/JRPROC.1948.226245

[19] Hitachi Chemical Co. Ultra small package tag by Hitachi Chemical, [Online] Cited 2018-11-20. Available at: http://www.hitachichem.co.jp/english/products/ppcm/014.html

[20] DU, M., XU, J., DING, X., et al. A low-profile wideband LTCC integrated circularly polarized helical antenna array for millimeter-wave applications. Radioengineering, 2018, vol. 27, no. 2, p. 455-462. DOI: $10.13164 /$ re.2018.0455

[21] FUJIMAKI, T., TOEDA, Y., HAMADA, M., et al. An electrically small on-chip antenna scaled down to one-twentyfifth by one-fiftieth of wavelength. In IEEE International Symposium on Antennas and Propagation and USNC/URSI National Radio Science Meeting. Boston (USA), 2018, p. 299-300. DOI: 10.1109/APUSNCURSINRSM.2018.8608514

[22] JANTARACHOTE, V., CHALERMWISUTKUL, S., SCHRAML, $\mathrm{K}$., et al. Comparison of meander line and NFRP miniaturization techniques for RFID on-chip antennas. In International Symposium on Antennas and Propagation (ISAP). Phuket (Thailand), 2017, p. 1-2. DOI: 10.1109/ISANP.2017.8228986

\section{About the Authors...}

Balazs MATOLCSY was born in Budapest, Hungary, in 1992. He received his B.Sc. and M.Sc. degree in Electrical Engineering at the Budapest University of Technology and Economics in 2015 and 2017, respectively. He joined the Department of Broadband Infocommunciation and Electromagnetic Theory in 2017 as a Ph.D. student. In 2018, he received a Short Term Research Scholarship from DAAD at the Technical University of Karlsruhe (KIT) in Germany. The main areas of his research are high-frequency RF simulation, antenna design, visible light communication and mixed signal electronics.

Attila ZOLOMY received his M.Sc. and Ph.D. degrees in 1994 and 2004, respectively, from the Budapest University of Technology and Economics in Electrical Engineering. He works currently at the university as a part-time research leader. He also works as a senior member at Silicon Laboratories Ltd. in Hungary. His main interests are microwave and millimeter wave circuits, small antennas, impedance matching and RF Integrated Circuits. 